\title{
ErbB4 Genotype Predicts Left Frontotemporal Structural Connectivity in Human Brain
}

\author{
Andreas Konrad*,', Goran Vucurevic ${ }^{2}$, Francesco Musso ${ }^{3}$, Peter Stoeter ${ }^{2}$, Norbert Dahmen' and \\ Georg Winterer ${ }^{3}$
}

'Department of Psychiatry and Psychotherapy, Johannes Gutenberg University, Mainz, Germany; ${ }^{2}$ Institute of Neuroradiology, Johannes Gutenberg University, Mainz, Germany; ${ }^{3}$ Department of Psychiatry, Heinrich-Heine University, Dusseldorf, Germany

\begin{abstract}
Diminished left frontotemporal connectivity is among the most frequently reported findings in schizophrenia and there is evidence that altered neuronal myelination may in part account for this deficit. Several investigations have suggested that variations of the genes that encode the Neuregulin I (NRGI)-ErbB4 receptor complex are associated with schizophrenia illness. As NRGI--ErbB4 has been implicated in neuronal myelination, we investigated with diffusion tensor imaging (DTI) whether fractional anisotropy (FA) — a putative measure of neuronal myelination - is predicted by a risk haplotype of the ErbB4 gene. The effects of the ErbB4 genotype were investigated in healthy subjects ( $N=59$; mean age: $22.6 \pm 1.8$ years). We also measured reaction time (RT) during a selective attention/ working memory paradigm (visual oddball). In the schizophrenia risk genotype group, we found lower FA in the temporal lobe white matter (WM) including frontotemporal fiber tracts, predominantly in the left hemisphere. RT was increased in the risk genotype group and correlated with FA in the affected brain region. As FA is considered to index structural integrity of WM, to which neuronal fiber myelination is contributing, our results suggest that variations of the ErbB4 genotype may confer risk for schizophrenia illness via its impact on left frontotemporal connectivity in human brain. Reliability and validity of the result is suggested by our observation that ( $I$ ) the FAgenotype association was not only obtained in the entire sample but also in both the split halves and (2) a statistical relationship was found among RT, genotype and FA.
\end{abstract}

Neuropsychopharmacology (2009) 34, 64I-650; doi: I 0. I038/npp.2008. I I2; published online 30 July 2008

Keywords: schizophrenia; myelin; diffusion tensor imaging; fractional anisotropy; structural connectivity; ErbB4

\section{INTRODUCTION}

The morphological correlates of schizophrenia are subtle, and range from a slight reduction in brain size to localized alterations in the morphology and molecular composition of specific neuronal, synaptic, and glial populations in several brain regions (Harrison and Weinberger, 2005). An increasing body of evidence from neuroimaging and postmortem studies also suggests that structural connectivity between brain regions is disturbed in schizophrenia and that altered myelination may - in part-account for this deficit (Konrad and Winterer, 2008). In particular, the frequently made observation in functional imaging and electrophysiological studies that left frontotemporal connectivity might be preferentially affected in schizophrenic patients and their relatives has recently obtained support from several microstructural imaging studies (Konrad and Winterer, 2008; Szeszko et al, 2008). However, it is currently

*Correspondence: Dr A Konrad, Department of Psychiatry and Psychotherapy, Johannes Gutenberg University, Untere Zahlbacher Str. 8, 55 131 Mainz, Germany, Tel: + 49 6131 173701, Fax: + 496131 176690, E-mail: andreas_konrad@gmx.de

Received 21 April 2008; revised 14 June 2008; accepted 2 July 2008 unknown what molecular mechanisms may account for the deficiency of structural connectivity in schizophrenia illness.

The notion that axonal myelination might be affected in schizophrenia is currently supported by post-mortem studies which have identified abnormalities in the myelin sheath (Uranova et al, 2001) and oligodendroglia (Hof et al, 2003). Decreased expression of several myelination-related genes in patients further supports the hypothesis of white matter (WM) abnormalities (Hakak et al, 2001). Indirect evidence also comes from recent genetic association studies involving an abnormal Neuregulin 1 (NRG1)-ErbB4 signaling pathway in risk for schizophrenia illness. NRG1 is involved in neural development, synapse formation, and synaptic plasticity by activating ErbB receptor tyrosine kinases (Anton et al, 2004; Liu et al, 2007; Thompson et al, 2007) but it has also been demonstrated to modulate myelination by regulating oligodendrocyte proliferation, differentiation, and myelination (Corfas et al, 2004; Taveggia et al, 2008). Among the ErbB proteins, ErbB4 is the best characterized for its functions in the CNS. ErbB4 isoforms exhibit distinct tissue-specific and brain-regionspecific patterns of expression (Mei and Xiong, 2008). For example, it has been demonstrated that the thalamocortical 
axon projection is regulated by NRG1 and ErbB4 (LopezBendito et al, 2006).

The NRG-1 is now thought of being one of the prime susceptibility genes for schizophrenia (Harrison and Law, 2006; Munafo et al, 2008). Among the ErbB receptor genes, ErbB4 is of particular interest and it has been chosen for analysis in this study because its coding gene ErbB4 has been reported to be a risk gene for schizophrenia illness. The ErbB4 gene spans $1.16 \mathrm{Mb}$ on chromosome 2q34, consists of 28 exons and encodes one of the four members of the mammalian ErbB family of transmembrane tyrosine kinases (Fox and Kornblum, 2005). To date, no coding mutations have been identified in the ErbB4 gene and all reported schizophrenia-associated risk single-nucleotide polymorphisms (SNPs) are noncoding intronic variants (Law et al, 2007). Law et al suggested that the risk ErbB4 SNPs may be functional through splicing regulation or may monitor functional elements within the gene associated with schizophrenia risk. A significant association with schizophrenia was shown in a large sample $(N=296)$ of schizophrenia families and control individuals $(N=370)$ for two three-SNPs ErbB4 haplotypes, one including rs7598440, rs839541, and rs839523 around exon 3 (Nicodemus et al, 2006). For this latter haplotype, the same group was able to confirm their findings in a sample of AfricanAmerican families (Nicodemus et al, 2006). Moreover, another association study of unrelated Ashkenazi schizophrenia patients $(N=59)$ and matched controls $(N=130)$ found similar results showing significant associations with 3 out of 19 SNPs along the ErbB4 gene from one linkage disequilibrium (LD) block: rs707284, rs839523, and rs7598440 (Silberberg et al, 2006). In addition, Silberberg et al (2006) reported that a schizophrenia risk haplotype around exon 3 (SNPs rs839523, rs707284, and rs7598440) is associated with altered expression of certain splice variants of the gene (CYT-1 isoform) in schizophrenia brain. This finding was confirmed by Law et al (2007) who found main effects for rs839523 as well as for rs707284, rs7598440, and a core-risk haplotype surrounding exon 3 on expression of the exon 26 (CYT-1) variant isoform of ErbB4 mRNA in hippocampus and dorsolateral prefrontal cortex. It has therefore been suggested that NRG1 may mediate its effects on schizophrenia susceptibility through functional interaction with ErbB4, and that genetic interaction between variants at the two loci together increases the genetic risk for schizophrenia (Norton et al, 2006). Norton et al examined 15 SNPs, revealed by mutation screening in schizophrenic patients, although evidence for association was shown for only 1 marker (rs7424835). Taken together, only a small number of ErbB4 association studies have been performed and some of the findings are controversial.

Two recent diffusion tensor imaging (DTI) studies have reported schizophrenia risk NRG1 genotype effects on WM microstructure in human brain (McIntosh et al, 2007; Winterer et al, 2008). These studies thus support the notion that variations of the NRG1 gene might indeed be relevant for neuronal myelination as fractional anisotropy (FA) is thought to be an index of the degree of neuronal myelination (Davis et al, 2003). In the present DTI study of young healthy Caucasian subjects $(N=59)$, we tested whether the previously identified schizophrenia risk SNPs from the haplotype around exon 3 of the ErbB4 gene are associated with FA. The scientific rationale of this study was that neuroimaging studies may help to elucidate the neurobiological mechanisms through which particular genes exert their effect on human brain. In addition, when endophenotypes (eg brain imaging measures) are used, it is expected that a gene effect, ie the effect size, is increased as compared to the effect on clinical phenotypes because biological phenotypes are thought to be closer to the genetic level (Winterer et al, 2005). Currently, a minimum sample size of $N=50$ is thought to be sufficient for genetic analyses of structural brain images, depending on the allele frequency (van Haren et al, 2008). For example, Szeszko et al (2005) examined the effect of the brain-derived neurotrophic factor val66met on hippocampal structural alterations in 44 subjects comparing $N=27 \mathrm{val} / \mathrm{val}$ homozygotes with $N=17 \mathrm{val} / \mathrm{met}$ heterozygotes. Zinkstok et al (2008) investigated whether variations in the catechol-Omethyltransferase gene are associated with brain anatomy in a sample of male schizophrenic patients of $N=51$ (genotype group sizes between 9 and 39 patients).

We investigated exclusively healthy subjects because it has been shown that antipsychotic drugs differentially regulate the expression of NRG1 and ErbB4 receptors in rat brain (Wang et al, 2008) and because prior studies have reported an association between FA and antipsychotic treatment (Minami et al, 2003; Eto et al, 2006; Kuroki et al, 2006) which both suggests that any investigation of ErbB 4 gene effects on brain structure could potentially be confounded by drug effects in schizophrenic patients. Moreover, schizophrenia is a complex polygenic disorder whereby each of multiple gene variations that are common in the general population is thought to contribute only a minor fraction to the overall risk (Harrison and Weinberger, 2005). Therefore, the effects of specific risk gene variations on brain structure can be investigated in the general population as well, ie in healthy subjects - even in the absence of overt behavioral abnormalities seen in patients - provided the employed phenotypic measure is related to the genetic risk for schizophrenia and that it is sensitive enough to detect genetic effects (Winterer et al, 2005). Therefore, our approach to investigate healthy subjects offers some advantages although it does not allow to study the interaction between the gene variation of interest and any other illness-related factors such as structural changes during the course of the illness or psychopathological characteristics. As part of our study, we also conducted a voxel-based morphometry (VBM) analysis in order to determine whether any observed FA genotype group differences are possibly due to indirect effects of brain volume differences. In order to assess the functional relevance of any given association, we also measured reaction time (RT) during oddball task conditions, which is a frequently applied endophenotype of selective attention/working memory to detect abnormal left frontotemporoparietal brain activation in subjects at risk for schizophrenia (Winterer et al, 2003; Javitt et al, 2008).

\section{METHODS}

\section{Subjects}

Healthy subjects $(N=59$; mean age $22.6 \pm 1.8$ years $)$ were investigated (Table 1). Only unrelated Caucasian subjects were included in the study. Participants were only 
Table I Demographic and Behavioral Data by Genotype Group rs839523

\begin{tabular}{lccc}
\hline rs839523 genotype & G/G & [A/G+A/A] & P-value \\
\hline Subjects (n) & 33 & 17 & \\
Age (years) & $22.6 \pm 1.8$ & $22.8 \pm 1.7$ & 0.72 \\
Male, female (n) & 17,16 & 8,9 & 0.77 \\
Education (years) & $13.8 \pm 3.2$ & $14.9 \pm 3.0$ & 0.28 \\
IQ & $118 \pm 11.6$ & $116 \pm 10.6$ & 0.66 \\
RT (ms) & $539 \pm 56$ & $497 \pm 45$ & 0.014 \\
RT variability (ms) & $100 \pm 25$ & $82 \pm 19$ & 0.017 \\
\hline
\end{tabular}

Abbreviations: IQ, intelligence quotient; RT, reaction time.

Subjects harbouring an $A$ allele are combined in one group [A/G+A/A]. Given are mean values and standard deviations in the sample with valid DTI data

$(N=50)$. Essentially identical results (not depicted) were found in the slightly larger VBM sample $(N=5 \mathrm{I})$ and for rs7598440, rs83954I and rs707284.

investigated if there was no evidence for any medical or neurological condition that could interfere with the purpose of the study, or if there was no history for any psychiatric DSM-IV axis I or axis II disorder including current or recent drug or alcohol abuse as assessed by a Structured Clinical Interview (First et al, 1995), a formal medical and neurological examination including urine toxicology for illegal drug abuse screening, routine blood tests, an urine investigation and a clinical EEG session. None of the investigated subject had family histories of schizophrenia or bipolar disorder. All subjects were right-handed. No statistically significant differences $(P<0.05)$ between genotype groups (rs839523, rs7598440, rs839541, rs707284) were found with regard to age and sex and intelligence quotient (IQ). IQ was assessed with the HAWIE-R (HamburgWechsler Intelligenztest) Scale (Tewes, 1991), which is largely equivalent with the full-scale Wechsler Adult Intelligence Scale-R (Kaplan et al, 1991). Only subjects with an IQ $>80$ were included in the study. Written informed consent was obtained from all study participants. The study was approved by the Ethics Committee of the Johannes Gutenberg University in Mainz (Germany).

\section{Image Acquisition}

Magnetic resonance imaging (MRI) scanning was performed with a $1.5 \mathrm{~T}$ Siemens Sonata ${ }^{\mathbb{R}}$ system at the Institute of Neuroradiology of the Johannes Gutenberg University, Mainz, Germany. An eight-channel head coil was used. Head movements were minimized by tightly fixating the head with cushions during the scanning procedure. After a localizer sequence for orientation, a high-resolution T1weighted MRI volume data set was acquired using a magnetization-prepared rapid gradient echo (MP-RAGE) sequence. The acquisition matrix was $256 \times 256,176$ slices were acquired with $1 \mathrm{~mm}$ slice thickness and a $15^{\circ}$ flip angle. Repetition time (TR) was $2860 \mathrm{~ms}$, echo time (TE) was $3.9 \mathrm{~ms}$.

DTI was conducted with echo-planar-imaging sequences. The images were acquired in six noncollinear diffusionsensitizing gradient directions with diffusion sensitivity $b=1000 \mathrm{~mm}^{2} / \mathrm{s}$ and one acquisition without diffusion encoding $\left(b=0 \mathrm{~mm}^{2} / \mathrm{s}\right)$. A generalized autocalibrating partially parallel acquisition reconstruction algorithm was used. Slices were positioned along the anterior commissure-posterior commissure line. The diffusion acquisition parameters were as follows: the acquisition matrix was $128 \times 128$ with a field of view of $192 \times 192 \mathrm{~mm}$ and a resolution of $1.5 \times 1.5 \times 2.0 \mathrm{~mm}^{3}$. Slice thickness was $2 \mathrm{~mm}$ and 64 axial slices were acquired to cover the whole brain without interslice gap. Other parameters were: $\mathrm{TR}=8000 \mathrm{~ms}, \mathrm{TE}=100 \mathrm{~ms}$. A total of 10 acquisitions were performed and averaged, the total duration of the DTI measurement was $20 \mathrm{~min}$.

\section{Image Preprocessing}

All scans were inspected visually. T1-weighted images from 8 subjects and DTI series from 9 subjects were excluded (due to gross motion artifacts or due to technical problems), DTI data sets from 50 subjects (Table 1) and T1 data sets from 51 subjects were included. Original MR diffusion, T1and T2-weighted images were registered in DICOM format and converted to ANALYZE format using MRIcro software (University of Nottingham, UK).

\section{Diffusion Tensor Imaging Analysis}

The T2-weighted images were normalized to the MNI (Montreal Neurological Institute) T2 template using SPM2 (statistical parametric mapping; Wellcome Department of Cognitive Neurology, London, UK) software implemented in MatLab 6.5 (Mathworks Inc., Sherborn, MA, USA). Identical normalization parameters were used for warping of the diffusion-weighted images such that each voxel represents the same part of the brain in every subject. For the calculation of FA maps, the FDT tool (FMRIB's diffusion toolbox) of the FSL software library (FMRIB's software library) was used. The obtained FA maps were then smoothed with a $6 \times 6 \times 6 \mathrm{~mm}$ FWHM Gaussian kernel to improve signal-to-noise ratio and normalization. The choice of using a $6 \mathrm{~mm}$ filter was based on evidence from imaging studies that the smoothing filter should be at least 2-3 times larger than the voxel size. Voxel-based FA contrast analysis was then done to compare genotype groups using general linear model (GLM) standard two sample unpaired $t$-test. Following established procedures (Shin et al, 2005; Szeszko et al, 2008), contrast maps were thresholded at a $P<0.001$ without correction for multiple comparisons and the extent threshold for significant clusters was set to 40 voxels.

We prepared an FA template according to the procedure described by Smith et al (2006), which was then overlaid with the statistically significant SPM clusters using MRIcro software for graphical presentation in neurological convention $(\mathrm{R}=\mathrm{R}$ ). The MRI atlas of human WM (Mori et al, 2005) was used for the identification of subcortical WM structures. The $t$-statistic of the peak voxel cluster, the cluster size, and the corresponding Brodman area and anatomical structures were determined (Mori et al, 2005). The MNI coordinates of the most significant voxel from the contrast analysis was used to calculate the mean FA values in the most significant 'peak voxel' together with six adjacent voxels in each subjects data set. This data were used to depict data in a scatterplot. 


\section{Voxel-based Morphometry Analysis}

Voxel-based morphometry was carried out with an optimized VBM protocol (Ashburner and Friston, 2000) using SPM5 software implemented in MatLab 7.1. The high-resolution T1weighted MRI data sets were first normalized to a standard template. The procedure then perfomed segmentation of the normalized images into gray matter (GM), WM, and cerebrospinal fluid (CSF). The segmented images were then smoothed with a $6 \mathrm{~mm}$ FWHM kernel. Voxel-wise comparisons of the normalized, segmented, and smoothed data were then performed between genotype groups with two contrasts (two sample unpaired $t$-test) to test for significant differences of local brain tissue concentration and volume.

\section{Biological Parametric Mapping}

Biological parametric mapping (BPM) combines information from different imaging modalities on a voxel-wise basis using the GLM (Casanova et al, 2007). After voxel-wise integration of imaging parameters, each voxel has a unique regression design that includes the value of each imaging modality for the voxel. In order to rule out important confounding genotype group differences in VBM measures and their effect on FA analyses in our data set, the analysis takes into account, separately for each voxel, the effect of a covariate image (VBM) on the image of interest (FA map).

The BMP toolbox (www.ansir.wfubmc.edu) is integrated in MatLab, the analysis was performed as described in detail by Casanova et al (2007). The resulting $\mathrm{T}$ maps generated in the BPM program were then exported to the SPM5 environment where they were thresholded at a $P<0.001$ (uncorrected) and overlaid on FA templates for graphical presentation of significant genotype group differences.

\section{Oddball Task}

To assess neurocognitive function, we measured RT during a visual oddball task as RT during oddball task conditions is known to be prolonged in schizophrenia patients while being associated with the degree of activation in frontotemporopariatal brain circuits (Frodl et al, 1998; Gur et al, 2007). Intraindividual RT variability, which reflects shortterm fluctuations in performance, was also measured because it was reported to be increased in patients with schizophrenia and because it was suggested to be a sensitive marker for the efficiency of top-down attentional control (Kaiser et al, 2008). Subjects were required to perform the oddball task right before the image acquisition. A total of 160 visual stimuli (40 targets, 120 nontargets, checkerboard reversal) were presented by means of a back-projection system onto a translucent screen using the 'Presentation' software package (Neurobehavioral Systems Inc.). Subjects were instructed to respond as quickly and accurate as possible to each stimulus (checkerboard) by pressing either the left button (nontarget) or the right button (target) with their index fingers. Stimuli were presented with a duration of $500 \mathrm{~ms}$ in counter-balanced and pseudo-randomized order at 'jittered' interstimulus intervals of $6000 \pm 500 \mathrm{~ms}$ between stimulus onsets, the total duration of the task was $960 \mathrm{~s}$. Motor responses (latencies) were recorded through a fiber optic response box.

\section{Genotyping}

DNA was obtained from venous blood using standard techniques. Four SNPs (rs7598440, rs839541, rs839523, and rs707284) from the ErbB4 gene around exon 3 (Figure 1)-that have been previously associated with schizophrenia (Nicodemus et al, 2006; Silberberg et al, 2006; Law et al, 2007) - were genotyped by the analysis of primer extension products generated from amplified genomic DNA using a Sequenom (San Diego, California) chip-based MALDI-TOF mass spectrometry platform. In brief, polymerase chain reaction (PCR) and extension reactions were designed using MassARRAY design software, and were carried out using $2.5 \mathrm{ng}$ of template DNA. Unincorporated nucleotides in the PCR product were deactivated using shrimp alkaline phosphatase. The primer extension products were then cleaned and spotted onto a SpectroChip with a massARRAY nanodispenser. The chips were scanned using a mass spectrometry workstation (MassARRAY compact analyzer; Sequenom) and the resulting spectra were analyzed and genotypes were determined using the Sequenom SpectroTYPER-RT software.

\section{Statistics}

Statistical analyses were conducted with Student's $t$-tests, Mann-Whitney $U$-tests or regression analysis as appropriate. As the FA values of the peak voxels were not normally distributed, we adopted Mann-Whitney $U$-tests for genotype group comparison. The ratio of explained variance of FA attributable to genotype to total variance was calculated as the ratio of the explained sum of squares to the total sum of squares. Tests for Hardy-Weinberg equilibrium (HWE), LD plots and pairwise LD values were calculated with the program haploview version 3.32 (Barrett et al, 2005).

To further increase statistical power, we combined the smaller homozygous genotype group with the heterozygous

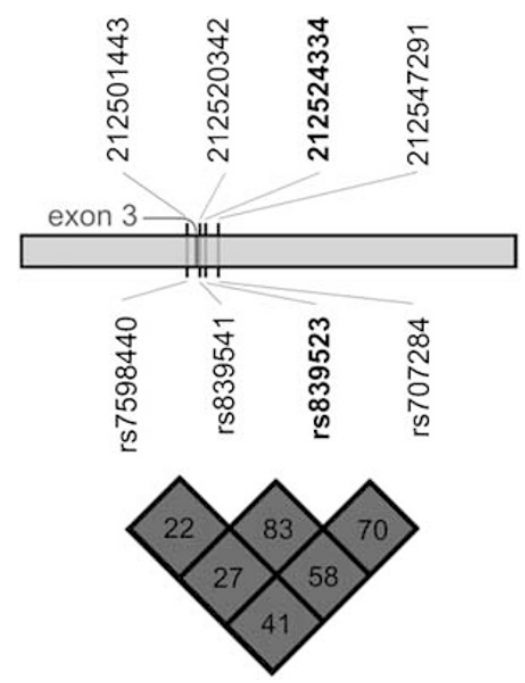

Figure I ErbB4 gene. (Top) ErbB4 gene with positions (bp) of reported single-nucleotide polymorphism (SNPs; Ensembl Genome Browser www.ensembl.org). (Bottom) Linkage disequilibrium (LD) plot ( $r^{2}$ values) for the four investigated SNPs in the present study in $N=59$ subjects. (haploview version 3.32) (Barrett et al, 2005). 
group depending on genotype distribution. An analogous procedure was chosen for analyzing haplotype effects. Sample sizes were too small to assess the putative modes of transmission. It is worth mentioning that the mode of transmission of schizophrenia risk conferring effects is unknown (Nicodemus et al, 2006; Silberberg et al, 2006).

\section{RESULTS}

\section{Genotype Distribution}

Allele frequencies of the four ErbB4 SNPs were similar to what has been reported previously (Nicodemus et al, 2006; Silberberg et al, 2006) and no significant deviations $(P<0.05)$ from HWE were observed (Table 2$)$. LD analysis between SNPs (Figure 1) showed high LD between the haplotype SNPs (rs7598440, rs839541, rs839523, and rs707284) which all have been reported to be associated with schizophrenia illness (Nicodemus et al, 2006; Silberberg et al, 2006; Law et al, 2007). For further analyses, we selected SNP rs839523 because it is tagging the schizophrenia risk haplotype block (Nicodemus et al, 2006; Silberberg et al, 2006) and because it was reported to show the highest association with schizophrenia of all investigated ErbB4 SNPs (Silberberg et al, 2006).

\section{Subject Parameters}

For all haplotype SNPs (rs7598440, rs839541, rs839523, and rs707284), we found no statistically significant differences $(P>0.05)$ between genotype groups $(\mathrm{G} / \mathrm{G}$ vs $[\mathrm{A} / \mathrm{G}+\mathrm{A} / \mathrm{A}])$ with regard to age, sex, education, and general intelligence (IQ) - neither in the VBM nor for the DTI sample (Table 1).

\section{SNP Genotype Effects on Structural Image Parameters}

Voxel-wise parametric FA contrast analyses of genotype groups revealed statistically significant group differences (thresholded at $P<0.001$, uncorrected) for SNP rs839523 (Figures 2 and 3 ) with a maximum in the left temporal lobe WM (peak MNI coordinates $-50,-18,-12 ; t=4.73$, cluster size $=271$ voxels) and in the right temporal lobe WM (52, $-4,-10 ; t=3.94,62$ voxels). In people homozygous for the

Table 2 Genotype Groups and Minor Allele Frequencies

\begin{tabular}{|c|c|c|c|c|c|}
\hline \multirow{2}{*}{$\begin{array}{l}\text { SNP } \\
\text { rs7598440 }\end{array}$} & \multicolumn{3}{|c|}{ Genotype groups $\mathbf{N}$} & \multirow[t]{2}{*}{ MAF } & \multirow[t]{2}{*}{$\begin{array}{l}\text { HWE } \\
\text { P-value }\end{array}$} \\
\hline & $\mathrm{T} / \mathrm{T}$ & $\mathrm{T} / \mathrm{C}$ & $\mathrm{C} / \mathrm{C}$ & & \\
\hline & 23 & 24 & 12 & 0.41 & 0.48 \\
\hline \multirow[t]{2}{*}{ rs83954l } & $\mathrm{T} / \mathrm{T}$ & $\mathrm{C} / \mathrm{T}$ & $\mathrm{C} / \mathrm{C}$ & & \\
\hline & 42 & 15 & 2 & 0.14 & 0.78 \\
\hline \multirow[t]{2}{*}{ rs839523 } & $\mathrm{G} / \mathrm{G}$ & G/A & $\mathrm{A} / \mathrm{A}$ & & \\
\hline & 40 & 16 & 3 & 0.19 & 0.72 \\
\hline \multirow[t]{2}{*}{ rs707284 } & $\mathrm{G} / \mathrm{G}$ & G/A & $\mathrm{A} / \mathrm{A}$ & & \\
\hline & 33 & 23 & 3 & 0.25 & 0.92 \\
\hline
\end{tabular}

Abbreviations: MAF, minor allele frequency; HWE, Hardy-Weinberg equilibrium; SNP, single-nucleotide polymorphism.

Genotype groups and allele frequencies with $N$ subjects for the four selected ErbB4 SNPs. schizophrenia risk allele $(G)$, FA was found to be significantly lower. The percentage of the variance in FA in the left temporal peak cluster that is attributable to genotype was $30.0 \%$. In the left temporal WM region, where the genotype-dependent peak maximum FA differences were observed, portions of several association tracts are closely aligned: inferior longitudinal fasciculus and superior longitudinal fasciculus and - in particular - a large part of two left frontotemporal connections, ie the inferior frontooccipital fasciculus (IFO) and uncinate fasciculus (Mori et al, 2005).

In the context of the main hypothesis, we performed an additional analysis of genotype effects on FA and regional brain volume for the other three SNPs (rs7598440, rs839541, and rs707284) of the risk haplotype around exon 3. Essentially identical results were found compared to rs839523 (Table 3). This was not unexpected given that the group compositions were largely similar because of the relatively high LDs between those three SNPs and rs839523 (Figure 1). As the selected SNPs were not independent because of the high LDs, we did not perform a Bonferroni correction for multiple testing. VBM analysis in all haplotype SNPs did not reveal any effects on regional brain volume (not depicted).

\section{Voxel-based Morphometry and Biological Parametric Mapping}

VBM parametric contrast analyses of the two SNP rs839523 genotype groups did not demonstrate any significant regional brain volume differences in the particular brain region or any other area in WM, GM, and CSF (thresholded at $P<0.001$, uncorrected).

The additional BPM voxel-wise covariate approach was performed for SNP rs839523 genotype groups to rule out important changes in VBM measures that failed to reach significance at the $P<0.001$ level. The results of this FA analysis demonstrate the essentially identical peak cluster in the left temporal lobe WM when accounting for VBM measures (peak MNI coordinates $-50,-18,-12 ; t=5.04$, cluster size $=192$ voxels; Figure 2 ). In addition, a significant cluster with FA reduction in the $G G$ genotype group was located in frontotemporal connections, corresponding to sections of the IFO and the uncinate fasciculus (peak MNI coordinates $-34,14,4 ; t=3.77$, cluster size $=57$ voxels; Figure 2). Though, no significant cluster in the right hemisphere was found in the BPM covariate analysis of FA maps.

\section{Split Half Analysis for rs839523}

Testing for the stability of the obtained results, two randomly selected subgroups (split halves) of the rs839523 genotype group were used for two separate group analyses. In both subgroups, we found the following genotype effects in left temporal WM: for the first subgroup $(N=25)$, peak MNI coordinates were $-56,-16,-8 ; t=3.99$ with cluster size $=51$ voxels. The corresponding values for the second subgroup $(N=25)$ were: $-52,-20,-10 ; t=3.88$, and cluster size $=13$ voxels. The peak cluster in both subgroups was located within the significant cluster of the entire sample, but it has to be mentioned that the peak cluster in 


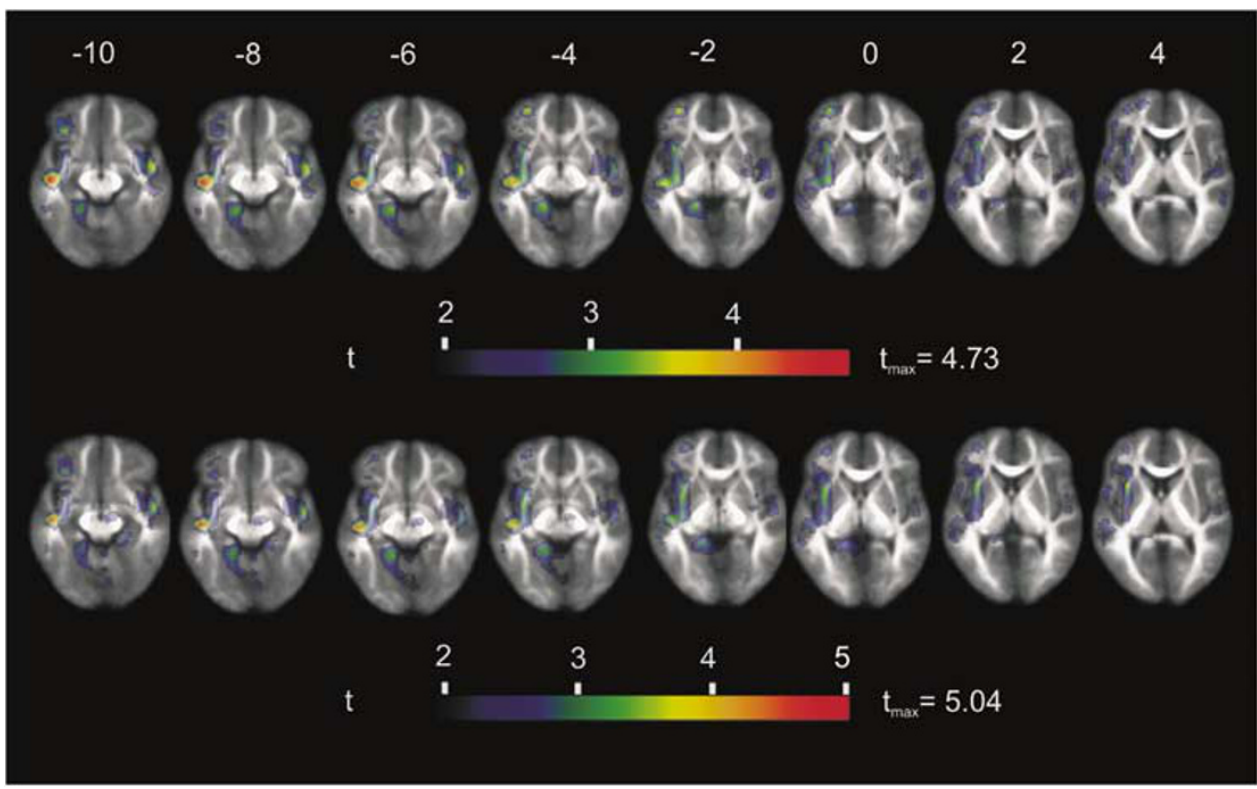

Figure 2 rs839523 genotype group effects (G/G vs A/G + A/A) on fractional anisotropy. (Top) Voxel-wise t-statistic genotype group FA contrast map overlaid on FA template on axial slices. Peak cluster in left temporal white matter (WM) (Montreal Neurological Institute, MNI coordinates, $x$ y z: $-50-I 8$ -12 ). Color scaling with $t$-value range from $t=1.68$ to $t_{\max }=4.73$. (Bottom) Voxel-wise covariate approach (Biological parametric mapping), which takes into account the effect of the VBM covariate image on the FA image of interest. Peak cluster in left temporal WM (MNI-coordinates, $x$ y z: - 50 - I8 - I2). Color scaling with $t$-value range from $t=1.68$ to $t_{\max }=5.0$. Notably, FA genotype effects in left frontotemporal connections are more prominent when accounting for VBM measures. Images are represented in neurological convention $(R=R)$. Given are the MNI $z$ coordinates for each slice. FA, fractional anisotropy; BPM, biological parametric mapping; VBM, voxel-based morphometry.
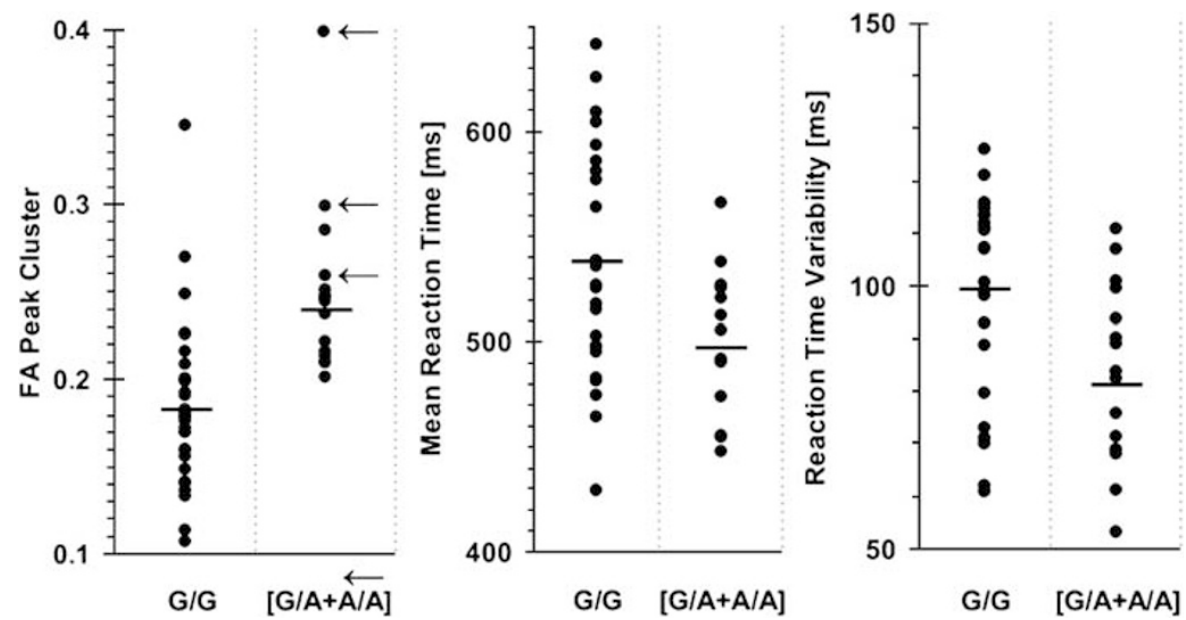

Figure 3 Scatterplots for rs839523 genotype group comparison (G/G vs [G/A + A/A]). (Left) rs839523 genotype group comparison of each subject's mean value $(N=50)$ of fractional anisotropy $(F A)$ in the 'peak voxel' together with six adjacent voxels in each direction. Genotype group differences are statistically significant (Mann-Whitney $U$-test, $Z=-4.31, P=1.63 \times 10^{-5}$ ). Arrows indicate A/A genotype carriers. (Middle) rs839523 genotype group comparison of each subject's mean reaction time (RT). Genotype group differences are statistically significant (Mann-Whitney $U$-test, $Z=-2.19, P=0.029$ ) (Right) rs839523 genotype group comparison of each subject's RT intrasubject variability. Genotype group differences are statistically significant (MannWhitney $U$-test, $Z=-2.51, P=0.012$ ).

the second subgroup was below the minimal cluster size of 40 voxels.

\section{Haplotype Effects on Structural Image Parameters}

In addition, we performed an analysis for the risk haplotype rs707284-rs758440-rs839523-rs839541 (G-T-G-T) comparing people homozygous for all four risk alleles $(N=18)$ with persons who were not homozygous for any of the risk alleles
$(N=14)$. Largely identical results to the above-mentioned single SNP analyses were found (Table 3). VBM analysis for haplotype effects did not reveal any effects on regional brain volume (not depicted).

\section{Genotype Effects on Reaction Time}

We observed a significant rs839523 genotype effect on RT (Mann-Whitney $U$-test: $\mathrm{Z}=-2.19, \quad P=0.029)$ and 
Table 3 Genotype Effects of SNPs and Haplotypes on Fractional Anisotropy

\begin{tabular}{|c|c|c|c|c|c|c|c|}
\hline & \multicolumn{2}{|c|}{ Group I } & \multicolumn{2}{|c|}{ Group 2} & \multirow{2}{*}{$\begin{array}{l}\text { Peak voxel } \\
\text { MNI }\end{array}$} & \multirow{2}{*}{$\begin{array}{c}\text { Cluster size } \\
\text { Voxel }\end{array}$} & \multirow{2}{*}{$\begin{array}{c}\text { Peak voxel } \\
t \text {-Value }\end{array}$} \\
\hline & Genotype & $\mathbf{N}$ & Genotype & $\mathbf{N}$ & & & \\
\hline rs758440 & $T / T$ & 19 & $\mathrm{C} / \mathrm{T}+\mathrm{C} / \mathrm{C}$ & 31 & $-48-20-8$ & NS & 2.81 \\
\hline rs83954I & $T / T$ & 35 & $\mathrm{C} / \mathrm{T}+\mathrm{C} / \mathrm{C}$ & 15 & $-52-16-10$ & 135 & 4.31 \\
\hline rs839523 (I half) & $\mathrm{G} / \mathrm{G}$ & 16 & $\mathrm{~A} / \mathrm{G}+\mathrm{A} / \mathrm{A}$ & 9 & $-52-20-10$ & $13^{\mathrm{a}}$ & 3.88 \\
\hline rs839523 (2 half) & $\mathrm{G} / \mathrm{G}$ & 17 & $\mathrm{~A} / \mathrm{G}+\mathrm{A} / \mathrm{A}$ & 8 & $-56-16-8$ & 51 & 3.99 \\
\hline \multirow[t]{2}{*}{ Haplotype (rs707284-rs758440-rs839523-rs83954I) } & G-T-G-T & 18 & Lower risk & 14 & $-48-20-8$ & 114 & 3.85 \\
\hline & G-T-G-T & 18 & All other & 32 & $-48-22-6$ & NS & 3.20 \\
\hline
\end{tabular}

Abbreviations: MNI, Montreal Neurological Institute; NS, not significant.

Given are peak voxel (thresholded at an uncorrected $P<0.00 \mathrm{I}$ ) values in left temporal white matter. The group I column contains subjects with a higher risk for schizophrenia than group 2. For rs839523, two randomly selected subgroups of each genotype group (split halves) were used for two separate group analyses. For haplotype analyses, comparisons are made between high-risk individuals (homozygous for G-T-G-T) and non-risk persons without any homozygous risk alleles or subjects with incomplete G-T-G-T risk allele sequence.

${ }^{a}$ For split halves and haplotype analysis, we include cluster $<40$ voxels even if not considered significant in main analysis.

intraindividual RT variability (Mann-Whitney $U$-test: $Z=-2.51, P=0.012$; Table 1; Figure 3). Mean RT and RT variability were significantly higher in the homozygous risk allele $(\mathrm{G} / \mathrm{G})$ group $(539 \pm 56 \mathrm{~ms})$ than in the combined heterozygous $(\mathrm{A} / \mathrm{G})$ and homozygous (A/A) group $(497 \pm 45 \mathrm{~ms})$. As expected, the task-related error rate did not differ significantly between groups, as it was very low $(=0.01)$ in both groups.

\section{Correlation of FA and RT/RT Variability}

Notably, individual FA values in the seven-voxel peak cluster in the left temporal WM were found to correlate with neurocognitive performance. Peak FA values were significantly correlated with RT $(r=-0.44, P=0.0036)$ and with RT variability $(r=0.38, P=0.015$; Figure 4$)$.

\section{DISCUSSION}

This is the first study suggesting that the ErbB4 gene affects subcortical microstructure in humans. Specifically, we found that subjects homozygous for the schizophrenia risk $G$ allele of SNP rs839523 (Nicodemus et al, 2006; Silberberg et al, 2006; Addington et al, 2007; Law et al, 2007) show decreased FA in the left temporal lobe WM-a brain region which contains frontotemporal fiber tracts but also fiber bundles that connect the parietooccipital and frontal cortex (Mori et al, 2005). SNP rs839523 was selected as a tagging SNP for the schizophrenia risk haplotype block, showing the highest association with schizophrenia of all investigated ErbB4 SNPs in the study of Silberberg et al (2006). Notably, abnormal activation and functional connectivity in left frontotemporoparietal brain regions is a well-known finding in schizophrenia patients and their relatives (Winterer et al, 2003). Accordingly, our findings may
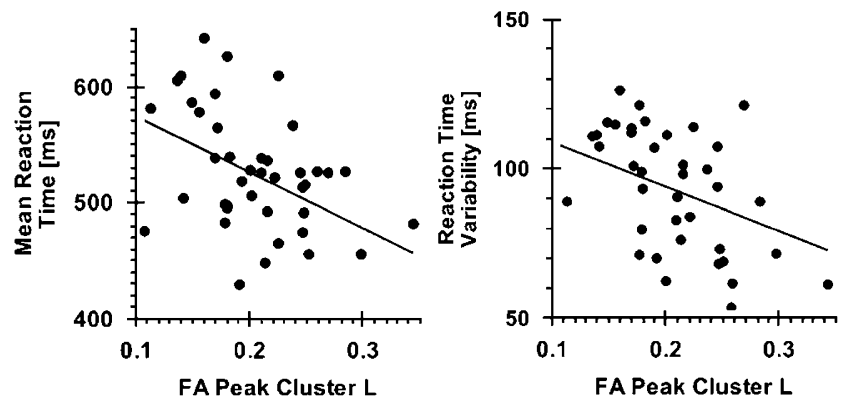

Figure 4 Regression analysis (left) between fractional anisotropy (FA) in the seven-voxel peak cluster in left temporal white matter (WM) and mean reaction time (Spearman's correlation coefficient $r=-0.44, P=0.0036$ ). Regression analysis (right) between FA in the seven-voxel peak cluster in left temporal WM and reaction time intrasubject variability (Spearman's correlation coefficient $r=-0.38, P=0.015$ ).

suggest that these functional deficits might be caused to some extent by structural variations of fiber tracts in this particular brain region involving the ErbB4 gene. It is therefore of interest that the functional relevance of ErbB4 - via its possible impact on the structural integrity of these fiber tracts - is further supported, ie externally validated, by our additional finding that FA was also related to RT which itself is frequently impaired in schizophrenia and which has been proposed to be an endophenotype of schizophrenia (Filbey et al, 2008). In this context, it is worth mentioning that several earlier studies have linked poor neurocognitive performance with reduced FA in frontotemporal fiber tracts (Szeszko et al, 2008). At the same time, our finding that the schizophrenia risk genotype predicts $\mathrm{RT}$ in the expected direction largely concurs with another study, which has reported ErbB4 genotype effects on neurocognitive measures in healthy controls (Nicodemus et al, 2006). The impact of schizophrenia risk NRG1 
genotypes on human WM has been shown in two DTI studies (McIntosh et al, 2007; Winterer et al, 2008). Therefore, our findings provide additional support for the notion that variations of the NRG1/ErbB4 signaling pathway might indeed be relevant for neuronal myelination. McIntosh et al (2007) demonstrated an effect of the SNP8NRG243177 genotype on the anterior limb of the internal capsule, whereas our group showed effects of the SNP8NRG221533 genotype from the same risk haplotype on medial frontal WM (Winterer et al, 2008). Abnormalities in these WM structures, as well as in frontotemporal fiber tracts, in schizophrenia have been shown in several DTI studies (Konrad and Winterer, 2008). Though, the impact of different NRG1/ErbB4 risk genotypes on different WM regions is currently difficult to interpret.

FA has been suggested to be a parameter for WM integrity but the biological determinants are not yet entirely understood (Schmithorst et al, 2008). However, an increase of FA has been found under nonpathological conditions during developmental myelination (Huppi and Dubois, 2006) and decreased FA values are well known in pathological conditions which impair myelination (Filippi et al, 2001). Even so, there is also evidence that the number and density of axon fibers and the coherence of these fibers influence FA (Papadakis et al, 1999). Thus, although it is intriguing to interpret our finding in a way such that the ErbB4 effect on FA and-by extension on RT-is due to an effect on neuronal myelination, other possibilities cannot be excluded with certainty at this point. Theoretically, it is also conceivable that indirect downstream effects on WM axon fibers, which may result - for instance-from gene-dependent abnormal synaptic input, account for our finding (Konrad and Winterer, 2008). In fact, this possibility does not appear entirely unlikely given that in animal brains, an ubiquitous expression of ErbB receptors has been demonstrated indicating that a variety of cell populations throughout the brain have the potential to respond to neuregulins in different ways. For example, Thompson et al (2007) reported that in frontal primate cortex, ErbB2, ErbB3, and ErbB4 antibodies immunostained neuronal soma and nuclei, the ErbB2 antibody also immunostained glia at the pial surface. Chong et al (2008) found that ErbB4 protein levels were significantly increased in prefrontal cortical cytoplasmic and nuclear fractions of schizophrenic patients relative to normal and to depressed subjects. On the other hand, ErbB3 and ErbB4 proteins were also localized to putative interstitial WM neurons whereas ErbB2 protein was found in glia. Roy et al (2007) recently reported of transgenic mice in which ErbB signaling was blocked in oligodendrocytes in vivo. They found that loss of all ErbB signaling (including ErbB2, 3 and 4) leads to changes in oligodendrocyte number and morphology, reduced myelin thickness, and slower conduction velocity in axons of central neurons. Others reported embryonic lethality in ErbB4 mouse mutants (Schmucker et al, 2003) and disturbed glial cell differentiation in ErbB4 knockout mice (Sardi et al, 2006). Thus, if the notion were correct that ErbB4 exerts its impact on FA via myelination, one might conclude that genetic susceptibility for schizophrenia via the ErbB4 gene could be mediated through the effect on neuronal myelination in frontotemporoparietal fiber tracts. In this context, a study of altered splice-variant expression in brain regions of schizophrenia patients is of particular interest: Law et al (2007) provided a direct link between disease-associated genetic variation including rs839523 and ErbB4 splice-variant mRNA expression levels in the human brain.

As expected and observed in many other genetic neuroimaging studies, the observed gene effect is subtle. It was, however, less expected that the observed gene effect is relatively circumscribed and limited to the left temporal region. This is because structural and functional imaging studies tend to find more widespread connectivity abnormalities in schizophrenia although most consistently in the left frontotemporal brain area. One might speculate that this region is particularly vulnerable to genetic variations during developmental myelination. A possible explanation could be that the age-dependent trajectory of myelination is dependent on ErbB4 genotype in this area due to the delayed and persistent myelination into the third decade of life in frontotemporal but not occipitoparietal fiber tracts (Yakovlev and Lecours, 1967; Paus et al, 1999), which indicates that oligodendrocyte regulation differs regionally. To some extent in line with notion idea would be the finding of Addington et al (2007) who observed in their longitudinal study of young schizophrenic patients that NRG1 risk allele carriers had different trajectories of change in brain lobar volumes, ie greater total GM and WM volume in childhood and a steeper rate of subsequent decline in volume into adolescence. Though, from the results of the current study, we are not able to suggest a developmental time point for the described effects on the brain structure.

Taken together, the findings of the present study indicate that ErbB4 gene variations may contribute its part to disturbed left frontotemporal connectivity in schizophrenia illness - possibly via an effect on myelination. The gene effect of a single risk SNP (rs839523) or haplotype, however, may play only a small role in total illness predisposition. Further replication studies - also involving clinical samples - are needed to confirm the results in our sample and to improve our understanding of the biological effects of the NRG1-Er6B4 signaling cascade in human brain.

\section{DISCLOSURE/CONFLICT OF INTEREST}

All authors report no competing interests.

\section{ACKNOWLEDGEMENTS}

We thank W. Hoeppner (Bioglobe GmbH, Hamburg, Germany) for genetic typing service. We also thank C. Schaeffner and M. Jahnke (Department of Psychiatry and Psychotherapy, Johannes Gutenberg University, Mainz, Germany) for technical and organizational support and S. Bauer and R. Radkowa for the neuropsychological investigations.

\section{REFERENCES}

Addington AM, Gornick MC, Shaw P, Seal J, Gogtay N, Greenstein $\mathrm{D}$ et al (2007). Neuregulin 1 (8p12) and childhood-onset schizophrenia: susceptibility haplotypes for diagnosis and brain developmental trajectories. Mol Psychiatry 12: 195-205. 
Anton ES, Ghashghaei HT, Weber JL, McCann C, Fischer TM, Cheung ID et al (2004). Receptor tyrosine kinase ErbB4 modulates neuroblast migration and placement in the adult forebrain. Nat Neurosci 7: 1319-1328.

Ashburner J, Friston KJ (2000). Voxel-based morphometry-the methods. Neuroimage 11: 805-821.

Barrett JC, Fry B, Maller J, Daly MJ (2005). Haploview: analysis and visualization of LD and haplotype maps. Bioinformatics 21: 263-265.

Casanova R, Srikanth R, Baer A, Laurienti PJ, Burdette JH, Hayasaka $S$ et al (2007). Biological parametric mapping: a statistical toolbox for multimodality brain image analysis. Neuroimage 34: 137-143.

Chong VZ, Thompson M, Beltaifa S, Webster MJ, Law AJ, Weickert CS (2008). Elevated neuregulin-1 and ErbB4 protein in the prefrontal cortex of schizophrenic patients. Schizophr Res 100: 270-280.

Corfas G, Roy K, Buxbaum JD (2004). Neuregulin 1-erbB signaling and the molecular/cellular basis of schizophrenia. Nat Neurosci 7: $575-580$.

Davis KL, Stewart DG, Friedman JI, Buchsbaum M, Harvey PD, Hof PR et al (2003). White matter changes in schizophrenia: evidence for myelin-related dysfunction. Arch Gen Psychiatry 60: 443-456.

Eto K, Eda K, Kanemoto S, Abe S (2006). The immunoglobulin-like domain is involved in interaction of Neuregulin1 with ErbB. Biochem Biophys Res Commun 350: 263-271.

Filbey FM, Toulopoulou T, Morris RG, McDonald C, Bramon E, Walshe $M$ et al (2008). Selective attention deficits reflect increased genetic vulnerability to schizophrenia. Schizophr Res 101: 169-175.

Filippi M, Cercignani M, Inglese M, Horsfield MA, Comi G (2001). Diffusion tensor magnetic resonance imaging in multiple sclerosis. Neurology 56: 304-311.

First MB, Spitzer RL, Gibbon M, Williams JBW (1995). The structural clinical interview for DSM-IV Axis I Disorders Research Version (SCID-I). American Psychiatric Press: New York.

Fox IJ, Kornblum HI (2005). Developmental profile of ErbB receptors in murine central nervous system: implications for functional interactions. J Neurosci Res 79: 584-597.

Frodl T, Meisenzahl EM, Gallinat J, Hegerl U, Moller HJ (1998). Markers from event-related potential subcomponents and reaction time for information processing dysfunction in schizophrenia. Eur Arch Psychiatry Clin Neurosci 248: 307-313.

Gur RE, Turetsky BI, Loughead J, Snyder W, Kohler C, Elliott M et al (2007). Visual attention circuitry in schizophrenia investigated with oddball event-related functional magnetic resonance imaging. Am J Psychiatry 164: 442-449.

Hakak Y, Walker JR, Li C, Wong WH, Davis KL, Buxbaum JD et al (2001). Genome-wide expression analysis reveals dysregulation of myelination-related genes in chronic schizophrenia. Proc Natl Acad Sci USA 98: 4746-4751.

Harrison PJ, Law AJ (2006). Neuregulin 1 and schizophrenia: genetics, gene expression, and neurobiology. Biol Psychiatry 60: 132-140.

Harrison PJ, Weinberger DR (2005). Schizophrenia genes, gene expression, and neuropathology: on the matter of their convergence. Mol Psychiatry 10: 40-68.

Hof PR, Haroutunian V, Friedrich VL, Byne W, Buitron C, Perl DP et al (2003). Loss and altered spatial distribution of oligodendrocytes in the superior frontal gyrus in schizophrenia. Biol Psychiatry 53: 1075-1085.

Huppi PS, Dubois J (2006). Diffusion tensor imaging of brain development. Semin Fetal Neonatal Med 11: 489-497.

Javitt DC, Spencer KM, Thaker GK, Winterer G, Hajos M (2008). Neurophysiological biomarkers for drug development in schizophrenia. Nat Rev Drug Discov 7: 68-83.
Kaiser S, Roth A, Rentrop M, Friederich HC, Bender S, Weisbrod M (2008). Intra-individual reaction time variability in schizophrenia, depression and borderline personality disorder. Brain Cogn 66: 73-82.

Kaplan E, Fein D, Morris R, Delis D (1991). WAIS-R as a Neuropsychological Instrument. The Psychological Corporation: New York.

Konrad A, Winterer G (2008). Disturbed structural connectivity in schizophrenia primary factor in pathology or epiphenomenon? Schizophr Bull 34: 72-92.

Kuroki N, Kubicki M, Nestor PG, Salisbury DF, Park HJ, Levitt JJ et al (2006). Fornix integrity and hippocampal volume in male schizophrenic patients. Biol Psychiatry 60: 22-31.

Law AJ, Kleinman JE, Weinberger DR, Weickert CS (2007). Disease-associated intronic variants in the ErbB4 gene are related to altered ErbB4 splice-variant expression in the brain in schizophrenia. Hum Mol Genet 16: 129-141.

Liu Y, Tao YM, Woo RS, Xiong WC, Mei L (2007). Stimulated ErbB4 internalization is necessary for neuregulin signaling in neurons. Biochem Biophys Res Commun 354: 505-510.

Lopez-Bendito G, Cautinat A, Sanchez JA, Bielle F, Flames N, Garratt AN et al (2006). Tangential neuronal migration controls axon guidance: a role for neuregulin-1 in thalamocortical axon navigation. Cell 125: 127-142.

McIntosh AM, Moorhead TW, Job D, Lymer GK, Munoz Maniega S, McKirdy J et al (2007). The effects of a neuregulin 1 variant on white matter density and integrity. Mol Psychiatry. (in press). (originally published online Oct. 9, 2007, at http://www.nature. $\mathrm{com} / \mathrm{mp} /$ journal/vaop/ncurrent/abs/4002103a.html).

Mei L, Xiong WC (2008). Neuregulin 1 in neural development, synaptic plasticity and schizophrenia. Nat Rev Neurosci 9: 437-452.

Minami T, Nobuhara K, Okugawa G, Takase K, Yoshida T, Sawada $S$ et al (2003). Diffusion tensor magnetic resonance imaging of disruption of regional white matter in schizophrenia. Neuropsychobiology 47: 141-145.

Mori S, Wakana S, Nagae-Poetscher LM, van Zijl PC (2005). MRI Atlas of Human White Matter. Elsevier Ltd: Oxford.

Munafo MR, Attwood AS, Flint J (2008). Neuregulin 1 genotype and schizophrenia. Schizophr Bull 34: 9-12.

Nicodemus KK, Luna A, Vakkalanka R, Goldberg T, Egan M, Straub RE et al (2006). Further evidence for association between ErbB4 and schizophrenia and influence on cognitive intermediate phenotypes in healthy controls. Mol Psychiatry 11: $1062-1065$

Norton N, Moskvina V, Morris DW, Bray NJ, Zammit S, Williams $\mathrm{NM}$ et al (2006). Evidence that interaction between neuregulin 1 and its receptor erbB4 increases susceptibility to schizophrenia. Am J Med Genet B Neuropsychiatr Genet 141: 96-101.

Papadakis NG, Xing D, Houston GC, Smith JM, Smith MI, James MF et al (1999). A study of rotationally invariant and symmetric indices of diffusion anisotropy. Magn Reson Imaging 17: 881-892.

Paus T, Zijdenbos A, Worsley K, Collins DL, Blumenthal J, Giedd JN et al (1999). Structural maturation of neural pathways in children and adolescents: in vivo study. Science 283: 1908-1911.

Roy K, Murtie JC, El-Khodor BF, Edgar N, Sardi SP, Hooks BM et al (2007). Loss of erbB signaling in oligodendrocytes alters myelin and dopaminergic function, a potential mechanism for neuropsychiatric disorders. Proc Natl Acad Sci USA 104: 8131-8136.

Sardi SP, Murtie J, Koirala S, Patten BA, Corfas G (2006). Presenilin-dependent ErbB4 nuclear signaling regulates the timing of astrogenesis in the developing brain. Cell 127: 185-197.

Schmithorst VJ, Holland SK, Dardzinski BJ (2008). Developmental differences in white matter architecture between boys and girls. Hum Brain Mapp 9: 696-710. 
Schmucker J, Ader M, Brockschnieder D, Brodarac A, Bartsch U, Riethmacher D (2003). ErbB3 is dispensable for oligodendrocyte development in vitro and in vivo. Glia 44: 67-75.

Shin YW, Kim DJ, Ha TH, Park HJ, Moon WJ, Chung EC et al (2005). Sex differences in the human corpus callosum: diffusion tensor imaging study. Neuroreport 16: 795-798.

Silberberg G, Darvasi A, Pinkas-Kramarski R, Navon R (2006). The involvement of ErbB4 with schizophrenia: association and expression studies. Am J Med Genet B Neuropsychiatr Genet 141: $142-148$.

Smith SM, Jenkinson M, Johansen-Berg H, Rueckert D, Nichols TE, Mackay CE et al (2006). Tract-based spatial statistics: voxelwise analysis of multi-subject diffusion data. Neuroimage 31: 1487-1505.

Szeszko PR, Lipsky R, Mentschel C, Robinson D, Gunduz-Bruce H, Sevy S et al (2005). Brain-derived neurotrophic factor val66met polymorphism and volume of the hippocampal formation. $\mathrm{Mol}$ Psychiatry 10: 631-636.

Szeszko PR, Robinson DG, Ashtari M, Vogel J, Betensky J, Sevy S et al (2008). Clinical and neuropsychological correlates of white matter abnormalities in recent onset schizophrenia. Neuropsychopharmacology 33: 976-984.

Taveggia C, Thaker P, Petrylak A, Caporaso GL, Toews A, Falls DL et al (2008). Type III neuregulin-1 promotes oligodendrocyte myelination. Glia 56: 284-293.

Tewes U (1991). Hamburg-Wechsler-Intelligenztest für Erwachsene. $H A W I E-R$. Huber: Bern.

Thompson M, Lauderdale S, Webster MJ, Chong VZ, McClintock B, Saunders R et al (2007). Widespread expression of ErbB2, ErbB3 and ErbB4 in non-human primate brain. Brain Res 1139: 95-109.
Uranova NA, Orlovskaia DD, Vikhreva OV, Zimina IS, Rakhmanova VI (2001). Morphometric study of ultrastructural changes in oligodendroglial cells in the postmortem brain in endogenous psychoses. Vestn Ross Akad Med Nauk 7: 42-48.

van Haren NE, Bakker SC, Kahn RS (2008). Genes and structural brain imaging in schizophrenia. Curr Opin Psychiatry 21: 161-167.

Wang XD, Su YA, Guo CM, Yang Y, Si TM (2008). Chronic antipsychotic drug administration alters the expression of neuregulin 1beta, ErbB2, ErbB3, and ErbB4 in the rat prefrontal cortex and hippocampus. Int J Neuropsychopharmacol 11: 553-561.

Winterer G, Coppola R, Egan MF, Goldberg TE, Weinberger DR (2003). Functional and effective frontotemporal connectivity and genetic risk for schizophrenia. Biol Psychiatry 54: 1181-1192.

Winterer G, Hariri AR, Goldman D, Weinberger DR (2005). Neuroimaging and human genetics. Int Rev Neurobiol 67: 325-383.

Winterer G, Konrad A, Vucurevic G, Musso F, Stoeter P, Dahmen $\mathrm{N}$ (2008). Association of $5^{\prime}$ end neuregulin-1 (NRG1) gene variation with subcortical medial frontal microstructure in humans. Neuroimage 40: 712-718.

Yakovlev PI, Lecours AR (1967). The myelogenetic cycles of regional maturation of the brain. In: Minkowski A (ed). Regional Development of the Brain in Early Life. Blackwell: Oxford. pp 3-65.

Zinkstok J, Schmitz N, van Amelsvoort T, Moeton M, Baas F, Linszen D (2008). Genetic variation in COMT and PRODH is associated with brain anatomy in patients with schizophrenia. Genes Brain Behav 7: 61-69. 